\title{
Multidisciplinary Management of Pancreatic Cancer
}

Presented by Margaret A. Tempero, MD

\begin{abstract}
Pancreatic cancer remains a vexing treatment challenge, with a cure rate that remains just $7 \%$. Two effective regimens-gemcitabine/nab-paclitaxel and FOLFIRINOX-have improved outcomes and are being used earlier in the disease. However, meaningful differences in outcomes may not be realized without novel strategies. Targeting of the immune system is an active area of research. (J Natl Compr Canc Netw 2015;13:700-702)
\end{abstract}

Among all malignancies, pancreatic cancer may be the most challenging. The relative dearth of treatment breakthroughs could change, as researchers are turning their focus on novel approaches, said Margaret A. Tempero, MD, Director of the University of California, San Francisco, Pancreas Center, Professor of Medicine at the UCSF Helen Diller Family Comprehensive Cancer Center, Chair of the Guidelines Panel and Editor-in-Chief of JNCCN_-Journal of the National Comprehensive Cancer Network.

In pancreatic cancer, $80 \%$ of patients are diagnosed with advanced unresectable disease; $80 \%$ experience relapse after resection and adjuvant therapy; and median overall survival (OS) of patients with untreated metastatic disease is only 3 months. The "cure rate" has not meaningfully changed over decades. And although mortality rates

Presented by Margaret A. Tempero, MD, Director of the University of California, San Francisco, Pancreas Center; Professor of Medicine at the UCSF Helen Diller Family Comprehensive Cancer Center, San Francisco, California; and Editor-in-Chief of JNCCN-Journal of the National Comprehensive Cancer Network.

Dr. Tempero has disclosed that she has served as a scientific advisor to AstraZeneca Pharmaceuticals LP, Asuragen, Bayer HealthCare, F. Hoffmann-LaRoche/Genentech, Myriad Genetic Labroartories, Inc., and NuCana BioMed and received grants or research support from Celgene Corporation.

Correspondence: Margaret A. Tempero, MD, UCSF Helen Diller Family Comprehensive Cancer Center, University of California, San Francisco, Mission Bay Campus / Mission Hall, 550 16th Street / 6th Floor / \#6541, San Francisco, CA 94143. E-mail: mtempero@medicine.ucsf.edu are declining for many other cancers, they are climbing in pancreatic cancer, which is poised to become the second, and possibly first, most common cause of cancer-related death.

"Even glioblastoma multiforme is a better diagnosis than pancreatic cancer," Dr. Tempero indicated, in a presentation at the NCCN 20th Annual Conference.

\section{Approach to Potentially Resectable Disease}

The 25-year history of adjuvant therapy can essentially be summarized as a half dozen clinical trials evaluating gemcitabine and 5-fluorouracil (FU), with and without radiation. Outcomes from these trials were largely similar, with median OS hovering around 22 months and 25\% to 30\% of patients dying of relapsed disease within 1 year of surgery.

Despite these less than ideal outcomes, adjuvant therapy is clearly superior to observation, she said, with the caveat that studies included highly selected populations. The same cannot be said for chemoradiation, the role of which has not been clearly established in clinical trials. The ongoing RTOG 0848 trial is examining the benefit of adding radiation after adjuvant gemcitabine monotherapy in 950 patients.

\section{Newer Approaches}

Adjuvant treatment has become more effective with the use of newer, presumably more active regimens, in particular FOLFIRINOX (5-fluorouracil, leucovorin, irinotecan, oxaliplatin) and gemcitabine plus nab-paclitaxel. Based on their benefit in the metastatic setting, these regimens are being incorporated into a number of recent adjuvant trials (Figure 1).

FOLFIRINOX is also increasingly being used as neoadjuvant treatment, especially to render borderline 


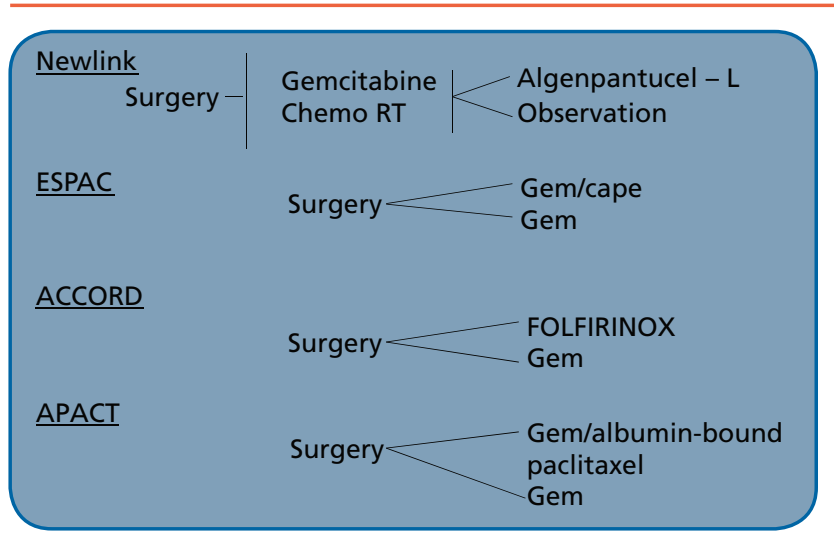

Figure 1 Adjuvant trials completed or in progress. Abbreviations: cape, capecitabine; ChemoRT, chemoradiation; ESPAC, the European Study Group for Pancreatic Cancer; Gem, gemcitabine.

resectable disease more resectable, but neoadjuvant studies have not yet established an OS benefit.

In brief, disease is considered clearly resectable when the superior mesenteric artery or common hepatic artery and superior mesenteric vein or portal vein are completely free from the tumor. The disease is clearly unresectable when disease encases those vessels. "Borderline" resectable is disease that closes in on those vessels ( $\leq 180$ degrees) but can still be resected, she explained.

There is particular interest in optimizing the treatment of borderline resectable tumors. Results are expected soon from A021101, a pilot study of neoadjuvant chemotherapy (FOLFIRINOX for 2 months) and chemoradiation followed by surgical resection and adjuvant gemcitabine. Early findings have been encouraging, Dr. Tempero indicated.

A larger trial in borderline resectable disease will evaluate neoadjuvant chemotherapy with and without radiation, followed by resection. "These studies will give us benchmarking data we have not had before," she said.

\section{Locally Advanced Pancreatic Adenocarcinoma}

In locally advanced disease, where resection is not possible, the "burning question" is the role of more effective combination chemotherapy and of radiotherapy after such chemotherapy.

French researchers evaluated the benefit of chemoradiation after 3 months of chemotherapy in patients with stage 3 to 4 disease in the GERCOR phase 2 and 3 trials. They found that discontinuation of chemotherapy followed by chemoradiation was more beneficial than continuing chemotherapy alone in nonprogressors. ${ }^{1}$ Median progression-free survival (PFS) was 10.8 versus 7.4 months $(P=.005)$, and median $O S$ was 15.0 and 11.7 months, respectively $(P=.0009)$.

At same time, however, 2 other trials in locally advanced disease reported conflicting results. The French 2000-01 FFCD/SFRO study evaluated intensive induction chemoradiotherapy followed by maintenance gemcitabine, versus gemcitabine alone and found OS to be shorter with chemoradiation (8.6 vs 13 months; $P=.03) .^{2}$ But a study by ECOG compared gemcitabine plus chemoradiation to gemcitabine alone and found OS to be better with chemoradiation (11.1 vs 9.2 months; $P=.017){ }^{3}$ Finally, the international phase 3 LAP07 study also concluded no benefit from chemoradiation, compared with gemcitabine alone. Median OS was 16.5 months in each arm. ${ }^{4}$

Based on current data, Dr. Tempero said, "If you are thinking about giving a patient with locally advanced disease gemcitabine monotherapy, and they do well on it, you cannot justify following this with radiation.... We don't know if you would get the same results with more effective combinations such as gemcitabine/nabpaclitaxel or FOLFIRINOX. Maybe."

An RTOG study is now evaluating the benefit of radiotherapy after gemcitabine/nab-paclitaxel.

\section{Effective Regimens in Metastatic Disease}

Dr. Tempero acknowledged that treatment in the metastatic setting is largely futile but reminded the audience that regimens identified as active in advanced disease are often successfully applied in the adjuvant setting.

A regimen that has shown a profound OS advantage in metastatic disease is FOLFIRINOX, which in the ACCORD trial reduced mortality by $43 \%(P<.0001)$, though the regimen can be difficult for patients. ${ }^{5}$ FOLFIRINOX can be made more tolerable by eliminating bolus 5 -FU, reducing doses, and using chemotherapy holidays. Patients whose disease responds well to this regimen can sometimes remain disease-free for months to years, according to Dr. Tempero, who advised, "Don't be afraid to stop treatment if the patient has a very good, deep response."

An OS benefit - a 28\% reduction in mortality $(P=.000015)$ — was also seen with gemcitabine/nab-paclitaxel in the MPACT study. ${ }^{6}$ Referring to the slightly smaller magnitude of benefit versus FOLFIRINOX, Dr. 
Tempero

Tempero cautioned that the patient population and degree of supportive care differed between the trials.

"It's not a contest. I am happy to have both these regimens to choose from," she commented. She often selects the regimen based on the patient's acceptance of the 2 different toxicity profiles.

As alternatives to FOLFIRINOX and gemcitabine/ nab-paclitaxel, the NCCN Guidelines list a number of options: gemcitabine/capecitabine for patients not fit enough for the preferred regimens; gemcitabine/cisplatin for patients with mutations in a DNA-damaging pathway (ie, BRCA); fixed-dose gemcitabine/docetaxel/ capecitabine; and gemcitabine/erlotinib.

\section{Focus on the Future}

To spur progress in pancreatic cancer, NCI held a "think tank" in October 2014 to promote promising areas of research. Two ideas were brought forward. One was to find a means of activating or "drugging" $R A S$, which remains a key "culprit" in tumor escape. The other idea was to target the tumor microenvironment, which in pancreatic cancer shows substantial desmoplasia (ie, the growth of dense connective tissue or stroma). Depletion of the stroma may allow anti-angiogenesis to be more effective. Other stromal-associated targets include activated stellate cells, interstitial fluid pressure, transforming growth factor-beta, $\mathrm{T}$ reg cells, and procarcinogenic immune cells.

Dr. Tempero is particularly excited about the idea of reprogramming the immune system. "Considering the genetic heterogeneity in cancer, our adaptive immunity may be our best asset in controlling disease progression," she suggested, indicating the variety of immune approaches being studied.

Addressing systemic inflammation through inhibition of JAK-STAT signaling could also prove effective. The JAK1/2 inhibitor ruxolitinib, approved in myelofibrosis, reduces levels of proinflammatory cytokines and was shown (with capecitabine) to significantly improve OS in pancreatic cancer patients with high $(>13 \mathrm{mg} / \mathrm{L})$ levels of $\mathrm{C}$-reactive protein (CRP) (HR, 0.47; P=.01).? The phase 3 JANUS trial will evaluate the drug in patients with pancreatic cancer and elevated CRP.

\section{References}

1. Huguet F, André $T$, Hammel $P$, et al. Impact of chemoradiotherapy after disease control with chemotherapy in locally advanced pancreatic adenocarcinoma in GERCOR phase II and III studies. J Clin Oncol 2007;20;25:326-331

2. Chauffert B, Mornex F, Bonnetain F, et al. Phase III trial comparing intensive induction chemoradiotherapy (60 Gy, infusional 5-FU and intermittent cisplatin) followed by maintenance gemcitabine with gemcitabine alone for locally advanced unresectable pancreatic cancer: definitive results of the 2000-01 FFCD/SFRO study. Ann Oncol 2008;19:1592-1599.

3. Loehrer PJ Sr, Feng Y, Cardenes H, et al. Gemcitabine alone versus gemcitabine plus radiotherapy in patients with locally advanced pancreatic cancer: an Eastern Cooperative Oncology Group trial. J Clin Oncol 2011;29:4105-4112.

4. Hammel P, Huguet F, Van Laethem J-L, et al. Comparison of chemoradiotherapy and chemotherapy in patients with a locally advanced pancreatic cancer controlled after 4 months of gemcitabine with or without erlotinib: final results of the international phase III LAP 07 study [abstract]. J Clin Oncol 2013;31 (Suppl):Abstract LBA4003.

5. Conroy $\mathrm{T}$, Desseigne F, Ychou $\mathrm{M}$, et al. FOLFIRINOX versus gemcitabine for metastatic pancreatic cancer. N Engl J Med 2011;364:1817-1825.

6. Von Hoff Dd, Ervin T, Arena FP, et al. Increased survival in pancreatic cancer with nab-paclitaxel plus gemcitabine. N Engl J Med 2013;369:1691703.

7. Hurwitz H, Uppal N, Wagner SA, et al. A randomized double-blind phase 2 study of ruxolitinib or placebo with capecitabine as second-line therapy in patients with metastatic pancreatic cancer [abstract].J Clin Oncol 2014;32(Suppl 5):Abstract 4000. 